\title{
A New Nematode Species (Seuratoidea, Cucullanidae) Parasitizing Parauchenipterus striatulus (Steindachner, 1876) (Pisces, Auchenipteridae) in Brazil
}

\author{
Narcisa IB Moreira ${ }^{+}$, Gilberto N Rocha, Hélio MA Costa ${ }^{\dagger}$ \\ Departamento de Parasitologia, Instituto de Ciências Biológicas, Universidade Federal de Minas Gerais, Caixa \\ Postal 486, 31270-901 Belo Horizonte, MG, Brasil
}

A new species of a cucullanid nematode is described, illustrated and compared with Cucullanus brevispiculus Moravec, Kohn \& Fernandes, 1993 and C. rhamphichthydis Moravec, Kohn \& Fernandes, 1997, two species previously reported as parasitizing freshwater fish in South America. The new species is characterized mainly by markedly short spicules, deirids and excretory pore situated posterior to the oesophago-intestinal junction, presence of strongly sclerotized plates in the oesophastome and oesophagus divided into two distinct portions.

Key words: nematodes - Cucullanus - new species - Parauchenipterus - freshwater fish - South America - Brazil

In this article a new species of Cucullanus Müller, 1777 is described and compared to other species of the genus which parasitize freshwater fish in South America.

\section{MATERIALS AND METHODS}

Fish were collected in lakes of the Rio Doce valley, State of Minas Gerais (19050'S, $\left.42^{\circ} 35^{\prime} \mathrm{W}\right)$, from May 1995 to July 1997. Specimens of a new cucullanid nematode of the genus Cucullanus were recovered from the intestine of the siluriform fish, Parauchenipterus striatulus (Steindachner, 1876), captured in Lago Central, a lake with an introduced ichthyofauna in Parque Estadual do Rio Doce.

The nematodes were washed in saline solution, fixed with warm AFA, preserved in $70^{\circ} \mathrm{Gl}$ alcohol and cleared with Aman's lactophenol. Drawings were made with the aid of a drawing tube, connected to a Wild bright field microscope. All measurements are given in millimeters.

\section{RESULTS \\ Cucullanus heliomartinsi $\mathrm{n} . \mathrm{sp}$}

(Figs 1- 4)

Description: medium-sized nematodes. Body slender, with smooth cuticle. Oral opening dorsoventrally elongate, surrounded by narrow mem-

\footnotetext{
$\overline{{ }^{+} \text {Corresponding author. Fax: }}+55-31-499.2970$. E-mail: brantnib@mono.icb.ufmg.br tDeceased in 6 January 1999 Received 19 April 1999 Accepted 22 November 1999
}

braneous collarette supported by 25 to 28 small triangular teeth. Pseudobuccal capsule (oesophastome) lined by a set of thickened sclerotized plates. Muscular oesophagus consisting of two distinct portions: anterior with sclerotized lining expanded anteriorly to form a pseudobuccal capsule, narrowing immediately below nerve ring; and posterior with a claviform aspect and strong muscular structure, opening into intestine through valves. Deirids and excretory pore situated posterior to oesophago-intestinal junction. Tail conical, with two phasmids.

Male (1 specimen): body 11.18 long and 0.216 wide. Oesophastome 0.081 long and 0.157 wide. Entire oesophagus 0.803 long, anterior portion 0.336 long and 0.184 wide and the posterior portion 0.461 long and 0.125 wide. Nerve ring 0.353 from anterior extremity. Deirids and excretory pore 0.933 and 0.881 from anterior extremity, respectively. Precloacal sucker well developed, 0.173 long and 0.544 from cloaca. Gubernaculum 0.093 long. Spicules equal, markedly short, slightly sclerotized, 0.280 long, correponding to $2.5 \%$ of the total body length, not reaching anteriorly half distance between cloacal aperture and precloacal sucker. Caudal papillae: 5 pairs preanal, 1 pair adanal and 5 pair postanal ( 3 subventral and 2 lateral). Tail smooth and pointed. Cloaca 0.312 from posterior extremity.

Female (1 specimen): body 17.45 long and 0.267 wide. Oesophastome 0.076 long and 0.163 wide. Total oesophagus 0.933 long, anterior portion 0.390 long and 0.217 wide, posterior portion 0.543 long and 0.179 wide. Nerve ring and deirids 0.380 and 1.004 from anterior extremity, respectively. Excretory pore not observed. Vulva postequatorial, 6.66 



Cucullanus heliomartinsi $\mathrm{n}$. sp. Fig. 1: anterior end of female, dorsal view. Fig. 2: region of deirids and excretory pore of male. Fig. 3: tail of male, lateral view. Fig. 4: tail of female. 
from posterior extremity. Vulvar lips prominent. Muscular vagina directed anteriorly. Uteri opposed. Immature eggs 0.047 long and 0.036 wide. Tail pointed. Anus 0.420 from posterior extremity. Phasmids 0.114 from tip of tail.

Type host: Parauchenipterus striatulus (Steindachner, 1876) (Auchenipteridae, Siluriformes).

Common names: "cumbaca", "cachaço".

Site of infection: intestine.

Type locality: Lago Central (Parque Estadual do Rio Doce, Minas Gerais, Brazil). Date of collection of holotype: December, 1995.

Etymology: the specific name is a tribute to the late Brazilian helminthologist Dr Hélio Martins de Araújo Costa.

Deposition of types: Instituto Oswaldo Cruz, Rio de Janeiro (Cat. no: 33.863A - holotype, and no: 33.863B - allotype)

\section{REMARKS}

According to Moravec et al. (1993), the genus Cucullanus Müller, 1777 includes several species that bear many similarities. The original descriptions of these are often poor, making comparisons between them difficult. Taking this and the endemicity of neotropical parasites of fish into account, the new species was compared to the Cucullanus species so far known from South American freshwater fish. According to Petter (1995) these are: C. pinnai Travassos, Artigas \& Pereira, 1928; $C$. zungaro Vaz \& Pereira, 1934; C. pauliceae Vaz \& Pereira, 1934 (syn. C. shubarti Travassos, 1947); C. mogi Travassos, 1947; C. colossomi DíazUngría, 1968; C. oswaldocruzi Santos, Vicente \& Jardim, 1979; C. grandistomis (Ferraz \& Thatcher, 1988); C. pseudoplatystomae Moravec, Kohn \& Fernandes, 1993; C. pimelodellae Moravec, Kohn \& Fernandes, 1993 and C. brevispiculus Moravec, Kohn \& Fernandes, 1993. C. patoi Fortes, Hoffmann \& Sarmento, 1992, C. fabregasi Fortes, Hoffmann \& Sarmento, 1993, C. riograndensis Fortes, Hoffmann \& Sarmento, 1993 and $C$. debacoi Sarmento, Fortes \& Hoffmann, 1995 were not included in Petter's survey and according to
Moravec et al. (1997) should be considered as species inquirendae. Moravec et al. (1997) described C. rhamphichthydis based on female specimens from a Brazilian host.

The main distinguishing characters of $C$. heliomartinsi $\mathrm{n}$. sp. are markedly short spicules that correspond to $2.5 \%$ of the total body length; deirids and excretory pore situated posterior to the oesophago-intestinal junction; marked sclerotizations in the oesophastome, and oesophagus divided in two distinct portions.

Although possessing a similar ratio of spicule/ body length to the new species, $C$. brevispiculus differs mainly from $C$. heliomartinsi in the position of deirids and the excretory pore, both located in the posterior half of the oesophagus.

Although males of $C$. rhamphichthydis are unknown, a comparison of the females was sufficient to distinguish it from the new species. In $C$. rhamphichthydis the deirids and the excretory pore are located very far from the posterior end of the oesophagus and the total length of the oesophagus corresponds to $10.93 \%$ of the body total length, whereas in $C$. heliomartinsi the proportion is $5.39 \%$.

This is the first report of a helminth parasite in fish of the genus Parauchenipterus.

\section{ACKNOWLEDGEMENTS}

To Drs Roberto Magalhães Pinto and Delir Corrêa Gomes MS Freire (Instituto Oswaldo Cruz), for their suggestions and helpful discussions.

\section{REFERENCES}

Moravec F, Kohn A, Fernandes BMM 1993. Nematode parasites of fishes of the Paraná River, Brazil. Part 2. Seuratoidea, Ascaridoidea, Habronematoidea and Acuarioidea. Folia Parasitol 40: 115-134.

Moravec F, Kohn A, Fernandes BMM 1997. New observations on seuratoid nematodes parasitic in fishes of the Paraná River, Brazil. Folia Parasitol 44: 209223.

Petter AJ 1995. Dichelyne moraveci n. sp., parasite de Pseudoplatystoma fasciatum et notes sur les Cucullanidae du Paraguay. Rev Suisse Zool 102: 769778. 